\title{
Fluoride Migration Catalysis Enables Simple, Stereoselective, and Iterative Glycosylation
}

Girish C. Sati, Joshua L. Martin, Yishu Xu, Tanmay Malakar, Paul M. Zimmerman, and John Montgomery*

Department of Chemistry, University of Michigan, Ann Arbor, MI 48109-1055, USA

*Corresponding author. Email: jmontg@umich.edu

\begin{abstract}
Challenges in the assembly of glycosidic bonds pose a bottleneck in enabling the remarkable promise of advances in the glycosciences. We report a strategy that applies unique features of electrophilic boron catalysts in addressing current limitations of methods in glycoside synthesis. The strategy utilizes glycosyl fluoride donors and silyl ether acceptors while tolerating the Lewis basic environment found in carbohydrates. The method allows a simple setup at room temperature while utilizing catalyst loadings as low as $0.5 \mathrm{~mol} \%$, and air- and moisture stable forms of the catalyst are found to be effective. These characteristics enable a wide array of glycosylation patterns to be accessed, including all four $\mathrm{C} 1-\mathrm{C} 2$ stereorelationships, and the method allows one-pot, iterative glycosylations to generate oligosaccharides directly from monosaccharide building blocks.
\end{abstract}

Main Text: Advances in the glycosciences present enormous promise in the understanding of disease and the development of strategies for improving human health.(1-3) The structural diversity and resulting biological properties of oligosaccharides and glycoconjugates are amplified by the stereochemical variations present within monosaccharide building blocks, the positioning (or absence) of oxygen and nitrogen functionality, and the multitude of possible points of connection between monosaccharide units. These features, paired with the multivalency of binding interactions, provide oligosaccharide chains with exquisite properties that govern many molecular recognition events in biology.

The inherent complexity of oligosaccharide preparation has been addressed by many innovative enzymatic and organic synthesis approaches. Enzymatic approaches are generally unmatched in preparative efficiency once developed and optimized,(4- $\sigma$ ) but organic synthesis approaches have the advantages of being more accessible to non-specialists and more easily and 
quickly adapted to diverse structural motifs. Despite the promise and potential generality of synthetic approaches, the development of rapid, robust, and operationally simple synthetic methods that are accessible to the broader biomedical research community remains as a critical need in glycoscience.(7) State-of-the-art chemical synthesis methods in carbohydrate chemistry often require specialized equipment and/or expert control of experimental variables including rigorous exclusion of moisture and air, precise temperature control, and handling of sensitive and unstable reagents. While many catalytic processes have been described, $(8)$ turnover numbers are often poor, and many of the most widely-used glycosylation methods require super-stoichiometric loadings of promotors. Additionally, the construction of each glycoside bond typically requires deprotection of a single hydroxyl, activation of an anomeric leaving group, stereoselective formation of the new glycoside bond, then deprotection of the next hydroxyl to be functionalized as the process is repeated. Innovative strategies have been developed for the iterative assembly of oligosaccharides through chemical approaches, $(9-15)$ but these methods often require technically challenging procedures with extensive variation of protecting groups, anomeric leaving groups, and/or activating reagents to impart stereoselectivity and regiocontrol. Automated methods provide the most promising non-enzymatic methods towards the programmed synthesis of oligosaccharides, (16-19) but experimentally simple, iterative synthesis through conventional, nonautomated methods without specialized equipment and procedures remains as a major gap in the field.

In this work, we address a number of the key challenges in chemical glycosylation. Specifically, goals of our work include the development of a rapid and simple approach that proceeds at room temperature, avoids the rigorous exclusion of moisture and air, provides flexible control of stereochemistry, and offers opportunities for iterative assembly of oligosaccharides using common building blocks. Our approach employs the use of glycosyl fluoride building blocks as the donor reagent and silyl ethers as the acceptor reagent. Glycosyl fluorides have long been recognized as a useful building block for glycosylations, and they are typically activated by stoichiometric promoters that sequester the fluoride leaving group. $(20,21)$ A potentially attractive but rarely employed protocol involves the coupling of glycosyl fluorides with silyl ethers, which allows glycoside bond formation along with a stable silicon fluoride byproduct.(22-26) The challenge in developing more practical versions of this process is the need for a stable and catalytically active Lewis acid to activate the glycosyl fluoride donor and a nucleophilic Lewis 
base to render cleavage of the stable silicon-oxygen bond of the glycosyl acceptor (Figure 1A). While the self-quenching characteristic of most Lewis acid-Lewis base pairs renders this approach untenable for efficient catalysis, we were attracted by the unique reactivity(27) and Frustrated Lewis Pair (FLP) characteristics(28-30) exhibited by $\mathrm{B}\left(\mathrm{C}_{6} \mathrm{~F}_{5}\right)_{3}$ (Figure 1B). Specifically, the equilibrium concentration of free Lewis acids and Lewis bases that coexist with structures of this type seemed ideally suited for glycosyl fluoride couplings with silyl ethers while avoiding deactivation of the Lewis acid by the Lewis-basic oxygen-rich nature of the carbohydrate building blocks. In support of this rationale, a unique fluoride-rebound mechanism was recently elucidated in $\mathrm{B}\left(\mathrm{C}_{6} \mathrm{~F}_{5}\right)_{3}$-catalyzed trifluoromethylations (Figure 1C), $(31,32)$ wherein the electrophilic triarylborane abstracts and then redelivers a fluoride ion in an efficient catalytic sequence. These observations in $\mathrm{B}\left(\mathrm{C}_{6} \mathrm{~F}_{5}\right)_{3}$ catalysis form the basis of a key hypothesis that electrophilic triarylborane catalysts might abstract the fluoride ion from a glycosyl donor, and then efficiently redeliver it as the Lewis base to activate the silyl ether component (Figure 1D). Furthermore, we anticipated that silyl structure variation in the acceptor reagent might be used as an approach to reverse stereoselectivity using inter- and intramolecular versions and also to sequence the reactivity of multiple hydroxyls in iterative one-pot couplings.

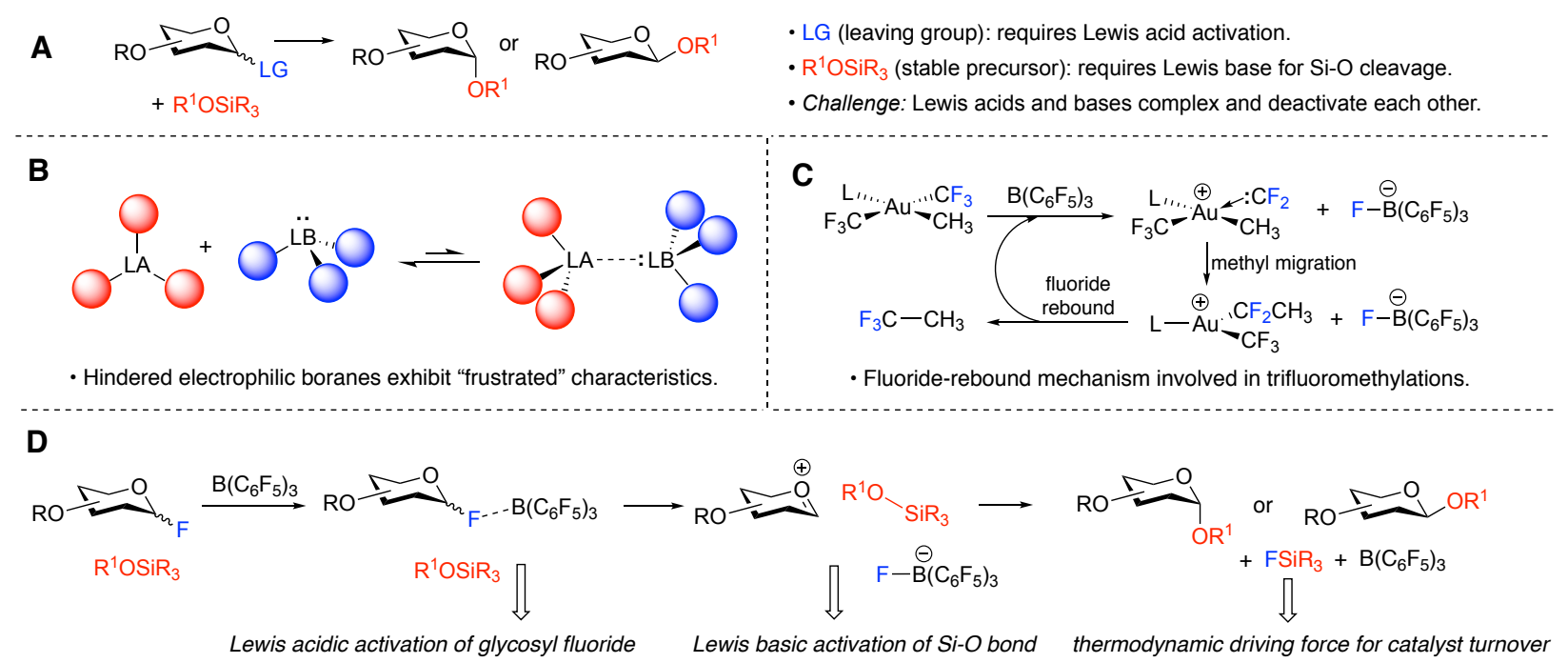

Fig. 1. Key design considerations of triarylborane-catalyzed glycosylations. (A) Challenges in direct utilization of silyl ethers in glycosylations. (B) Key characteristic of Frustrated Lewis Pairs (FLPs). (C) Fluoride-rebound mechanism in trifluoromethylations using $\mathrm{B}\left(\mathrm{C}_{6} \mathrm{~F}_{5}\right)_{3}$. (D) Central hypothesis for the design of triarylborane-catalyzed coupling of glycosyl fluorides and silyl ethers. 
The feasibility of this approach was examined in glycosidic couplings to install trans C1$\mathrm{C} 2$ linkages using $\mathrm{C} 2, \mathrm{C} 3, \mathrm{C} 4$ and $\mathrm{C} 6$ acceptors across a range of monosaccharide combinations with various protecting groups (Figure 2). Utilizing $5 \mathrm{~mol} \% \mathrm{~B}\left(\mathrm{C}_{6} \mathrm{~F}_{5}\right)_{3}$ at $\mathrm{rt}$ in toluene or dichloromethane solvent, high-yielding, rapid, and highly stereoselective glycosylations were observed. Acetate protection at the $\mathrm{C} 2$ position was typically employed since this protecting group is well established to direct the formation of C1-C2-trans glycosides through neighboring group participation. Glycosylations of "armed" glycosyl donors that possess benzyl protecting groups at C3 to C6 were exceedingly efficient, often proceeding to completion in 5-10 min using equimolar quantities of the monosaccharide donor and acceptor, providing access to $\alpha$-mannoside (1-6) products. As the examples show, protected mannose fluoride donors underwent efficient couplings with a range of sugars, including the equatorial and axial C3- and C4-hydroxyls of glucose and galactose. Notably, cyclic acetal protecting groups (3) and more hindered silyl ethers (1b, 1c, and 6) were cleanly tolerated in the method, allowing for selective deprotection and further synthetic elaboration. $n$-Pentenyl glycosides (4) were also tolerated, which thus allow subsequent couplings with orthogonal activation conditions. Protected glucosyl fluoride donors were also employed with $\mathrm{C} 2$-acetate direction, providing access to $\beta$-glucoside products (8-11). This stereochemical series was effective with hindered C2, C3, and C4 hydroxyls of the acceptor. Commonly employed 2azidosugars underwent efficient coupling (12) with poor stereocontrol (as expected in the absence of a C2-directing group), and the corresponding 2-phthalimido sugars were highly efficient with an improved 5:1 diastereoselectivity favoring the $\beta$-anomer (13). "Disarmed" glycosyl donors, which are deactivated by inductively withdrawing peracetylation, are also effective with this method as the above examples illustrate $(\mathbf{5 b}, \mathbf{7}$, and $\mathbf{8 b})$, although reaction times at $\mathrm{rt}$ typically extend to several hours. A silyl ether of phenol participated efficiently, producing the $O$-aryl glycoside product in high yield as a single diastereomer (14). Disaccharide products such as 1a may be deacetylated and then further utilized as the acceptor to allow efficient formation of trisaccharide products (15). Additionally, free hydroxyls can be used as the acceptor (i.e. example 2) although reactions are slower and proceed in reduced yield compared with the trimethylsilyl ether acceptor. 


$$
\mathrm{RO}_{\mathrm{F}_{\mathrm{F}}}+\mathrm{R}^{1} \mathrm{OSiMe}_{3} \stackrel{\mathrm{B}\left(\mathrm{C}_{6} \mathrm{~F}_{5}\right)_{3}(5 \mathrm{~mol} \%)}{\text { toluene, } \mathrm{rt}} \text { RO } \stackrel{\mathrm{OR}^{1}}{\longrightarrow} \text { or }
$$

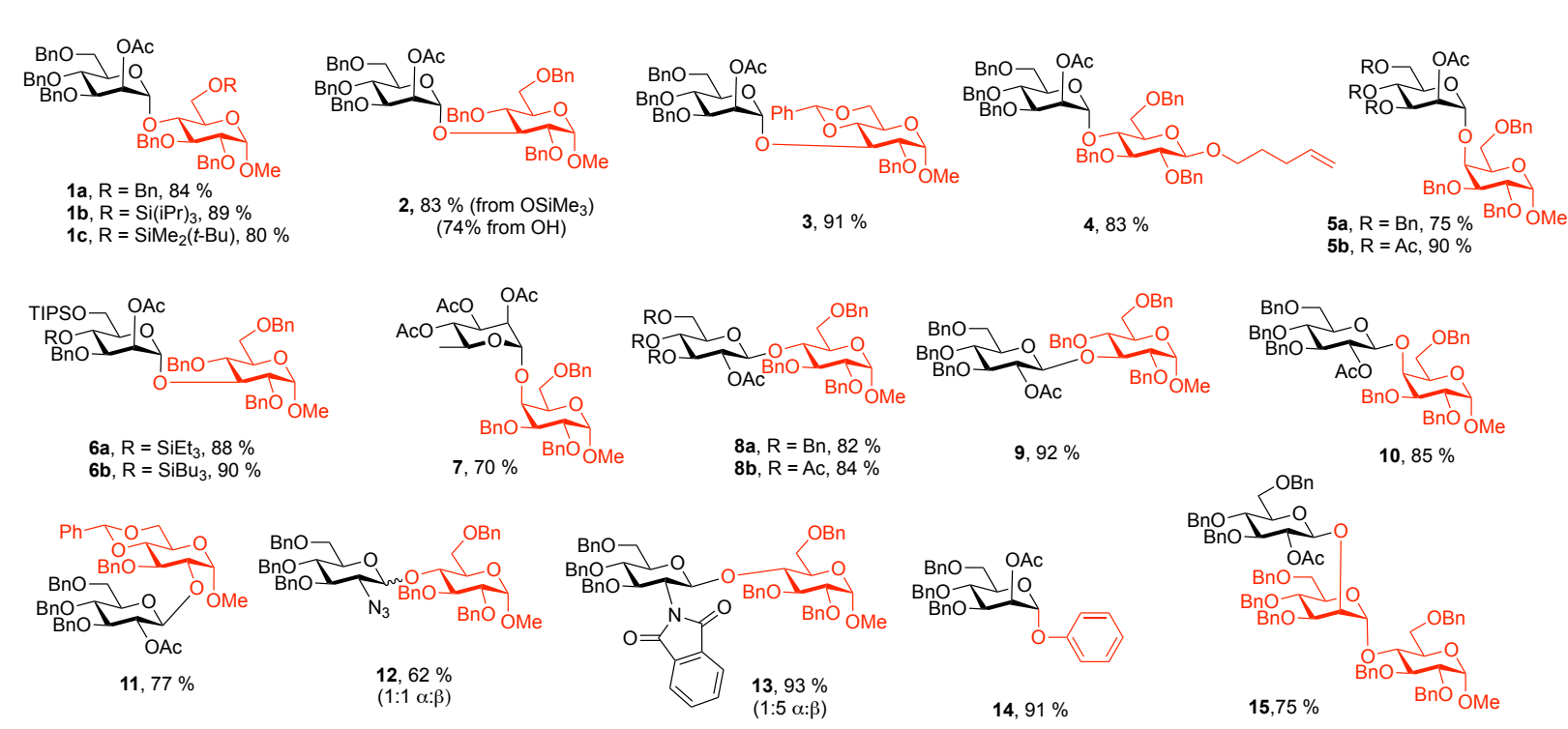

Fig. 2. Triarylborane-catalyzed couplings of glycosyl fluorides and silyl ethers. Reactions were conducted with 1.0 equiv of glycosyl fluoride and 1.1 equiv of silyl ether using $5 \mathrm{~mol} \%$ $\mathrm{B}\left(\mathrm{C}_{6} \mathrm{~F}_{5}\right)_{3}$ in toluene at room temperature. Isolated yields are reported. A single diastereomer of each product was observed unless otherwise noted.

The parent catalyst $\mathrm{B}\left(\mathrm{C}_{6} \mathrm{~F}_{5}\right)_{3}$ itself is hygroscopic and is typically stored and handled under inert atmosphere conditions. With an eye towards improving experimental ease and efficiency, we sought to explore the extent to which catalyst loadings can be lowered, and also to examine more stable catalyst variations (Figure 3A). While the above studies employed $5 \mathrm{~mol} \%$ catalyst, in a representative case (1a), catalyst loadings could be lowered to $0.5 \mathrm{~mol} \%$, which is markedly below the loading levels reported in other catalytic glycosylation methods. A number of catalyst structure modifications were examined, but most promising was the direct utilization of the simple hydrate $\mathrm{H}_{2} \mathrm{O} \cdot \mathrm{B}\left(\mathrm{C}_{6} \mathrm{~F}_{5}\right)_{3} .(27,33)$ This species is stable to air and moisture, and is an easily handled white crystalline, highly soluble catalyst that enables a simple benchtop setup for the catalytic glycosylations. The activity of this catalyst is lower than observed for the commercial form of $\mathrm{B}\left(\mathrm{C}_{6} \mathrm{~F}_{5}\right)_{3}$, but it still possesses sufficient reactivity for fast, room temperature reactions at the $5 \mathrm{~mol}$ $\%$ level in a $1 \mathrm{~h}$ reaction time. Using the hydrate $\mathrm{H}_{2} \mathrm{O} \cdot \mathrm{B}\left(\mathrm{C}_{6} \mathrm{~F}_{5}\right)_{3}$, a preparative experiment conducted on a $1.0 \mathrm{~g}$ scale afforded the mannose $\alpha-4$ glucose linkage of 1a in high yield (Figure 3A). To further benchmark the operational ease of this method, the preparation of 1a was 
conducted with $\mathrm{H}_{2} \mathrm{O} \cdot \mathrm{B}\left(\mathrm{C}_{6} \mathrm{~F}_{5}\right)_{3}$ as catalyst, using unpurified ACS grade solvents in an open flask at room temperature. Under these conditions, glycoside 1a was obtained in 77\% isolated yield. Other common Lewis acids such $\mathrm{BF}_{3} \bullet \mathrm{Et}_{2} \mathrm{O}$ and TMSOTf are also competent in promoting the glycosylations, but $\mathrm{B}\left(\mathrm{C}_{6} \mathrm{~F}_{5}\right)_{3}$ and its hydrate were the simplest and most effective across the range of applications described in this study. The very high catalytic activity of $\mathrm{B}\left(\mathrm{C}_{6} \mathrm{~F}_{5}\right)_{3}$, paired with its crystallinity, ease of handling, water tolerance, and tunable structure make this the optimum catalyst choice for these studies.

While the mechanistic hypothesis for this work is outlined above, computational experiments(34) have been carried out to better understand the features of this catalytic system (Figure 3B). Using a mannose $\alpha$-fluoride substrate, $\mathrm{B}\left(\mathrm{C}_{6} \mathrm{~F}_{5}\right)_{3}$ rapidly abstracts fluoride via transition state TS1 from intermediate $\mathbf{1 6}$ to provide the charged glycosyl donor 17 with stabilization from the neighboring $\mathrm{C} 2$-acetate. The lowest energy pathway for glycosylation involves formation of adduct $\mathbf{1 8}$ followed by rate-determining addition of the silyl ether to the charged intermediate to form 19 via transition state TS2, and then the $\mathrm{F}-\mathrm{B}\left(\mathrm{C}_{6} \mathrm{~F}_{5}\right)_{3}$ anion rapidly delivers fluoride to produce the $\alpha$-mannoside product and the $\mathrm{Me}_{3} \mathrm{Si}-\mathrm{F}$ byproduct while releasing the active $\mathrm{B}\left(\mathrm{C}_{6} \mathrm{~F}_{5}\right)_{3}$ catalyst.

The above methods allow intermolecular couplings to provide highly selective access to the $\mathrm{C} 1$-C2-trans $\beta$-glucoside and $\alpha$-mannoside stereochemical relationships. Silicon linkages have alternatively been used, typically with thioglycosides or anomeric sulfoxides, in an intramolecular aglycone delivery (IAD) strategy to access C1-C2-cis glycosides. (35, 36) While the C1-C2 trans glycosides $\mathbf{2 0}$ and $\mathbf{2 2}$ are available by the above approach, alternatively assembling a silicon connection between $\mathrm{C} 2$ of the donor and the desired reactive hydroxyl on the acceptor (in this case C6), the IAD strategy using glycosyl fluorides with $\mathrm{B}\left(\mathrm{C}_{6} \mathrm{~F}_{5}\right)_{3}$ catalysis provides an effective way to access the $\mathrm{C} 1-\mathrm{C} 2$-cis $\alpha$-glucoside 21 and $\beta$-mannoside 23 stereochemical relationships (Figure 3C and 3D). This versatile strategy of coupling glycosyl fluorides of glucose or mannose with silyl ethers in either an inter- or intramolecular sense allows all four C1-C2 stereochemical relationships to be accessed in high yield with exceptional stereocontrol, without requiring modification of the protecting group pattern on the $\mathrm{C} 3, \mathrm{C} 4$, and C6 hydroxyls of the glycosyl donor reagent. 

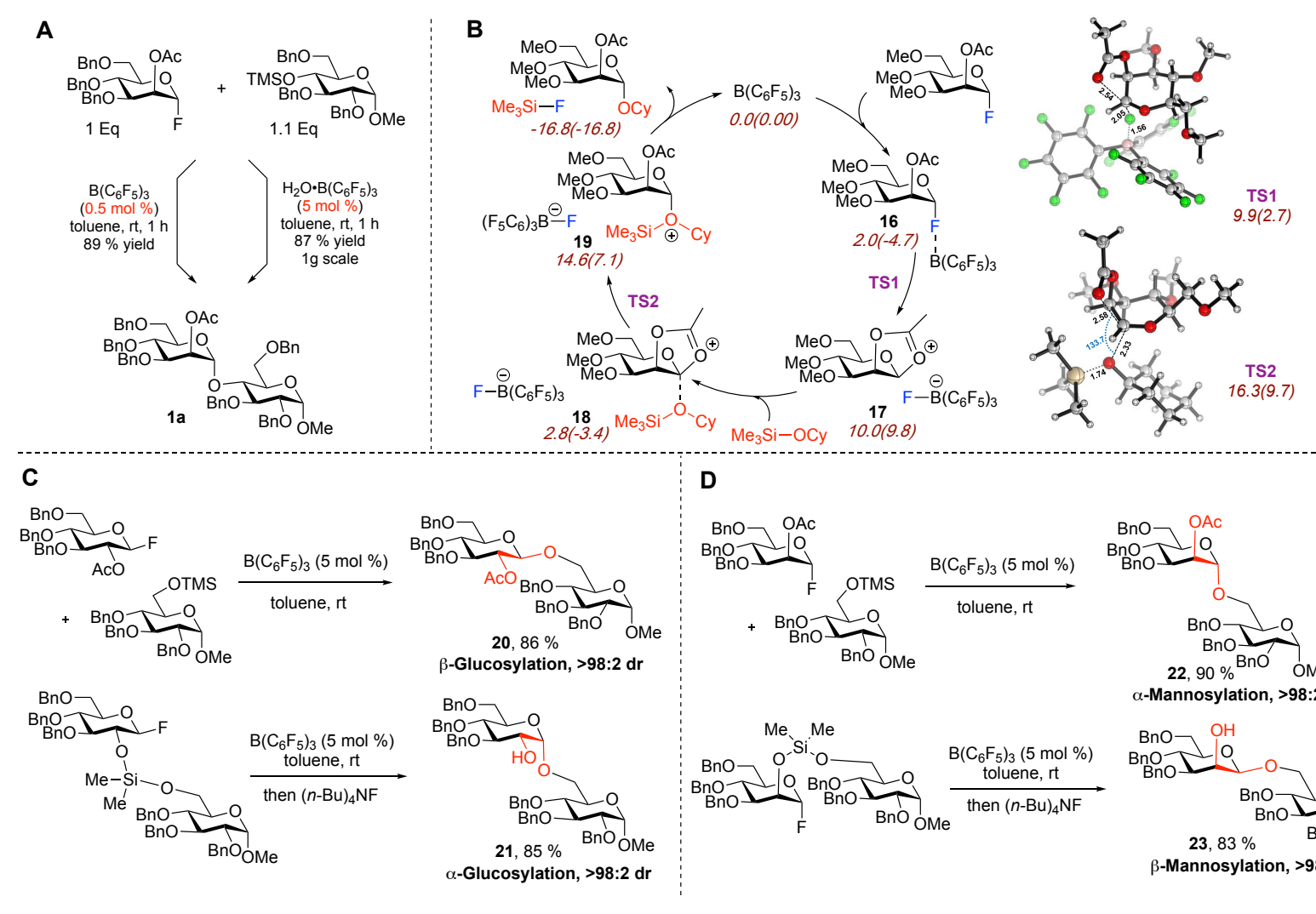

Fig. 3 Evaluation of operational ease, mechanism, and stereoselectivity. (A) Optimization of catalyst turnover and pre-catalyst stability and ease of handling. (B) Computational study of reaction mechanism using $\omega$ B97X-D3/def2-TZVP level of theory/method. Solvent phase Gibbs free energy (enthalpy in parentheses) values referenced against the starting value for the substrates and catalyst are in $\mathrm{kcal} / \mathrm{mol}$. Optimized bond distances and angles are in $\AA$ and degrees respectively. (C) Stereoselective synthesis of $\beta$ - and $\alpha$-glucosides. (D) Stereoselective synthesis of $\alpha$ - and $\beta$ - mannosides.

Finally, we sought to develop iterative, one-pot glycosylations using multiple monosaccharide building blocks. Such an approach through chemical catalysis requires several demanding features, namely fast couplings without an excess of any of the monosaccharide building blocks, paired with a strategy for differentiating the glycosyl donor leaving groups and the acceptor nucleophilic hydroxyls. The reactivity and stoichiometry requirements are met with this triarylborane-catalyzed method, and we envisioned that the glycosyl fluorides could be differentiated through order of addition while the hydroxyls could be differentiated by varying the steric hindrance of the silicon protecting group. To explore this objective, we first examined three component couplings of monosaccharides 24-26 (Figure 4). Initial coupling of 24 and 25a or 25b 
proceeded through completely regioselective glycosylation of the $\mathrm{C} 4$ trimethylsilyl-protected hydroxyl of $\mathbf{2 5 a} / \mathbf{b}$. Injecting 26 after $30 \mathrm{~min}$ led to the completely regio- and stereoselective production of $\mathbf{3 0 a}$ or $\mathbf{3 0 b}$ through glycosylation of the C6 triisopropylsilyl-protected hydroxyl. Simply changing the order of addition (initial addition of 25a and $\mathbf{2 6}$ followed by addition of 24) led to the completely regio- and stereoselective production of 30c. Similarly, monosaccharides 2729 were utilized to allow production of trisaccharides $\mathbf{3 1 a} / \mathbf{b}$ with complete regio- and stereocontrol by adding 27 and $\mathbf{2 8}$ first, followed by addition of $\mathbf{2 9}$ after $30 \mathrm{~min}$.

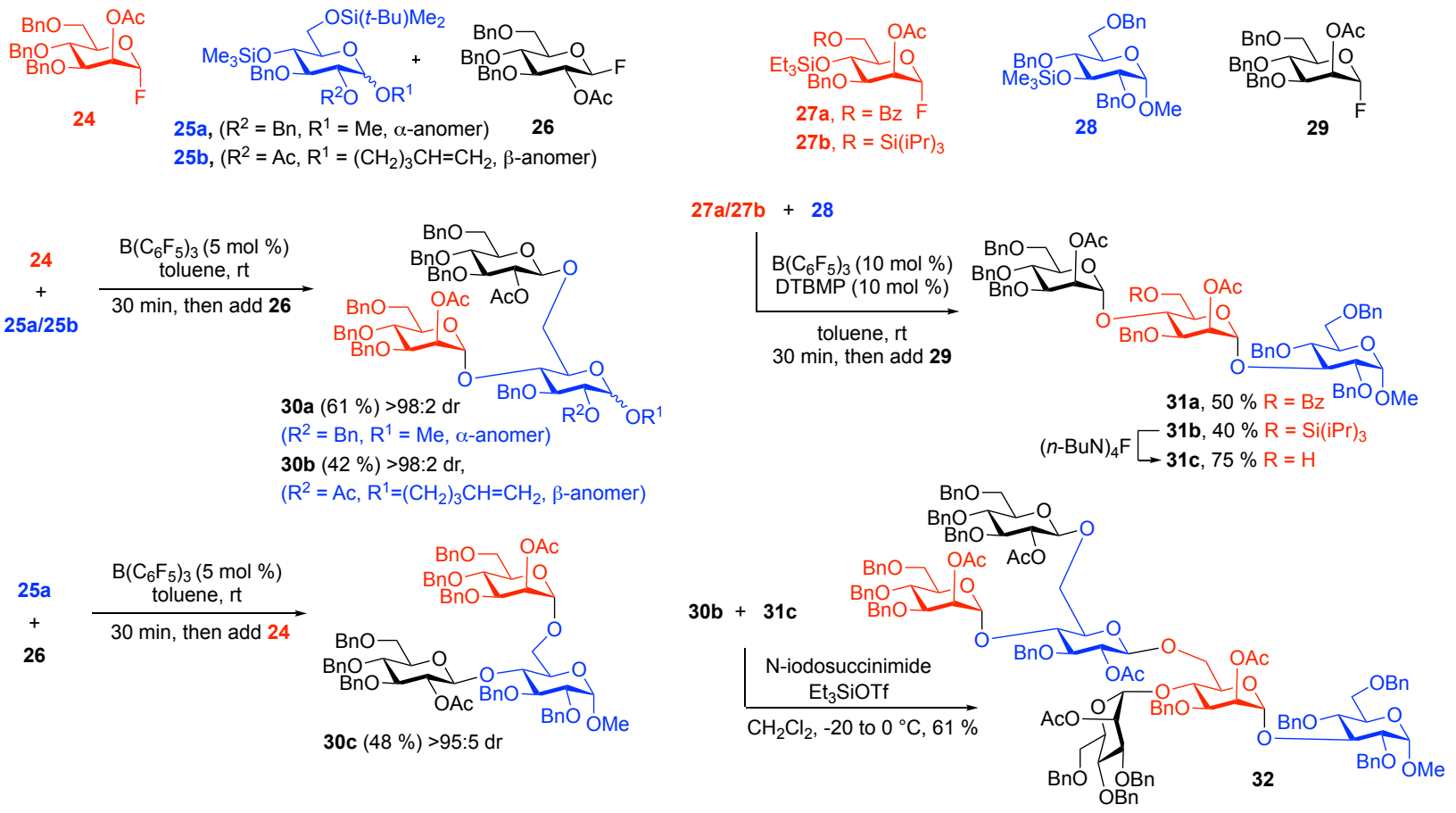

Fig. 4. Iterative Assembly of Oligosaccharides. $\mathrm{B}\left(\mathrm{C}_{6} \mathrm{~F}_{5}\right)_{3}$-catalyzed reactions were conducted using $5 \mathrm{~mol} \% \mathrm{~B}\left(\mathrm{C}_{6} \mathrm{~F}_{5}\right)_{3}$ in toluene at room temperature. Isolated yields are reported and a single diastereomer of products was obtained.

These examples (Figure 4) illustrate that the relative reactivity of hydroxyls is exclusively controlled by silicon protecting group rather than by the innate reactivity of the acceptor employed. Utilizing two or more silicon units on the acceptor enables installation of branched patterns (i.e. $\mathbf{2 5 a} / \mathbf{b}$ ), whereas utilizing one or more silicon units and a glycosyl fluoride on the same monosaccharide enables installation of linear patterns (i.e. 27a/b). Notably, $n$-pentenyl glycosides are tolerated (30b) and can be used in subsequent couplings using $N$-iodosuccinimide/Et ${ }_{3}$ SiOTf. $_{\text {iOn }}$ This approach allows hexasaccharide $\mathbf{3 2}$ to be produced as a single regio- and stereoisomer from 
monosaccharide precursors 24-29 in three linear steps (four total steps: three glycosylations and a single deprotection). The convergency of this approach demonstrates the practical advantages of the multicomponent nature of the triarylborane-catalyzed glycosylations.

In summary, a boron-catalyzed glycosylation method involving the coupling of glycosyl fluorides and silyl ethers addresses a number of key challenges in the synthesis of glycosidic bonds. The preparative ease of the method allows simple reaction setup using a commercially-available catalyst, without requiring specialized equipment or expertise. Reactions are often completed in minutes at room temperature across a range of glycosyl donor and acceptor classes. Through the combination of inter- and intramolecular strategies, all four of the possible C1-C2 stereochemical relationships may be accessed by this method. Computational studies illustrate a fluoride migration catalysis mechanism where the triarylborane catalyst abstracts fluoride from the donor reagent and then delivers fluoride to silicon as the glycoside product and active catalyst are released. The relative reactivity of hydroxyls on the acceptor substrate are governed by silane structure rather than by the innate reactivity present in the substrates. This feature enables the regioselective, iterative assembly of oligosaccharides in a simple, one-pot operation utilizing monosaccharide precursors. The above features position this method as attractive for addressing a number of synthetic challenges in glycoscience.

\section{References}

1. C. R. Bertozzi, L. L. Kiessling, Chemical Glycobiology. Science 291, 2357-2364 (2001).

2. P. Agre, C. Bertozzi, M. Bissell, K. P. Campbell, R. D. Cummings, U. R. Desai, M. Estes, T. Flotte, G. Fogleman, F. Gage, D. Ginsburg, J. I. Gordon, G. Hart, V. Hascall, L. Kiessling, S. Kornfeld, J. Lowe, J. Magnani, L. K. Mahal, R. Medzhitov, R. J. Roberts, R. Sackstein, R. Sarkar, R. Schnaar, N. Schwartz, A. Varki, D. Walt, I. Weissman, Training the Next Generation of Biomedical Investigators in Glycosciences. J. Clin. Invest. 126, 405-408 (2016).

3. L. L. Kiessling, Chemistry-Driven Glycoscience. Bioorg. Med. Chem. 26, 5229-5238 (2018).

4. L. Q. Wen, G. Edmunds, C. Gibbons, J. B. Zhang, M. R. Gadi, H. L. Zhu, J. Q. Fang, X. W. Liu, Y. Kong, P. G. Wang, Toward Automated Enzymatic Synthesis of Oligosaccharides. Chem. Rev. 118, 8151-8187 (2018).

5. A. R. Prudden, L. Liu, C. J. Capicciotti, M. A. Wolfert, S. Wang, Z. W. Gao, L. Meng, K. W. Moremen, G. J. Boons, Synthesis of Asymmetrical Multiantennary Human Milk Oligosaccharides. Proc. Natl. Acad. Sci. U. S. A. 114, 6954-6959 (2017). 
6. L. Krasnova, C. H. Wong, Oligosaccharide Synthesis and Translational Innovation. J. Am. Chem. Soc. 141, 3735-3754 (2019).

7. https://commonfund.nih.gov/Glycoscience

8. M. M. Nielsen, C. M. Pedersen, Catalytic Glycosylations in Oligosaccharide Synthesis. Chem. Rev. 118, 8285-8358 (2018).

9. S. S. Kulkarni, C. C. Wang, N. M. Sabbavarapu, A. R. Podilapu, P. H. Liao, S. C. Hung, "One-Pot" Protection, Glycosylation, and Protection-Glycosylation Strategies of Carbohydrates. Chem. Rev. 118, 8025-8104 (2018).

10. Z. Y. Zhang, I. R. Ollmann, X. S. Ye, R. Wischnat, T. Baasov, C. H. Wong, Programmable One-Pot Oligosaccharide Synthesis. J. Am. Chem. Soc. 121, 734-753 (1999).

11. D. R. Mootoo, P. Konradsson, U. Udodong, B. Fraser-Reid, Armed and Disarmed n-Pentenyl Glycosides in Saccharide Couplings Leading to Oligosaccharides. J. Am. Chem. Soc. 110, 5583-5584 (1988).

12. S. Raghavan, D. Kahne, A One-Step Synthesis of the Ciclamycin Trisaccharide. J. Am. Chem. Soc. 115, 1580-1581 (1993).

13. S. V. Ley, H. W. M. Priepke, A Facile One-Pot Synthesis of a Trisaccharide Unit from the Common Polysaccharide Antigen of Group-B Streptococci Using Cyclohexane-1,2-Diacetal (CDA) Protected Rhamnosides. Angew. Chem. Int. Ed. 33, 2292-2294 (1994).

14. X. F. Huang, L. J. Huang, H. S. Wang, X. S. Ye, Iterative One-Pot Synthesis of Oligosaccharides. Angew. Chem. Int. Ed. 43, 5221-5224 (2004).

15. K. M. Hoang, N. R. Lees, S. B. Herzon, Programmable Synthesis of 2-Deoxyglycosides. $J$. Am. Chem. Soc. 141, 8098-8103 (2019).

16. O. J. Plante, E. R. Palmacci, P. H. Seeberger, Automated Solid-Phase Synthesis of Oligosaccharides. Science 291, 1523-1527 (2001).

17. P. H. Seeberger, The Logic of Automated Glycan Assembly. Acc. Chem. Res. 48, 1450-1463 (2015).

18. M. Panza, S. G. Pistorio, K. J. Stine, A. V. Demchenko, Automated Chemical Oligosaccharide Synthesis: Novel Approach to Traditional Challenges. Chem. Rev. 118, 8105-8150 (2018).

19. S.-L. Tang, N. L. B. Pohl, Automated Fluorous-Assisted Solution-Phase Synthesis of $\beta-1,2-$, 1,3-, and 1,6-Mannan Oligomers. Carbohydr. Res. 430, 8-15 (2016).

20. M. Shimizu, H. Togo, M. Yokoyama, Chemistry of Glycosyl Fluorides. Synthesis, 799-822 (1998).

21. T. J. Wadzinski, A. Steinauer, L. N. Hie, G. Pelletier, A. Schepartz, S. J. Miller, Rapid Phenolic O-Glycosylation of Small Molecules and Complex Unprotected Peptides in Aqueous Solvent. Nat. Chem. 10, 644-652 (2018).

22. S. Hashimoto, M. Hayashi, R. Noyori, Glycosylation Using Glucopyranosyl Fluorides and Silicon-Based Catalysts - Solvent Dependency of the Stereoselection. Tetrahedron Lett. 25, 1379-1382 (1984). 
23. W. S. Kim, S. Hosono, H. Sasai, M. Shibasaki, Rare-Earth Perchlorate Catalyzed Glycosidation of Glycosyl Fluorides with Trimethylsilyl Ethers. Tetrahedron Lett. 36, 44434446 (1995).

24. T. Zhu, G. J. Boons, A Two Directional Glycosylation Strategy for the Convergent Assembly of Oligosaccharides. Tetrahedron Lett. 39, 2187-2190 (1998).

25. K. C. Nicolaou, A. Chucholowski, R. E. Dolle, J. L. Randall, Reactions of Glycosyl Fluorides - Synthesis of O-Glycosides, S-Glycosides, and N-Glycosides. J. Chem. Soc. Chem. Commun., 1155-1156 (1984).

26. H. Kunz, W. Sager, Stereoselective Glycosylation of Alcohols and Silyl Ethers Using Glycosyl Fluorides and Boron-Trifluoride Etherate. Helv. Chim. Acta 68, 283-287 (1985).

27. W. E. Piers, A. J. V. Marwitz, L. G. Mercier, Mechanistic Aspects of Bond Activation with Perfluoroarylboranes. Inorg. Chem. 50, 12252-12262 (2011).

28. D. W. Stephan, The Broadening Reach of Frustrated Lewis Pair Chemistry. Science 354, 1248 (2016).

29. C. B. Caputo, D. W. Stephan, Activation of Alkyl C-F Bonds by $B\left(\mathrm{C}_{6} \mathrm{~F}_{5}\right)_{3}$ : Stoichiometric and Catalytic Transformations. Organometallics 31, 27-30 (2012).

30. K. Ishihara, N. Hanaki, M. Funahashi, M. Miyata, H. Yamamoto, Tris(Pentafluorophenyl)Boron as an Efficient, Air-Stable, and Water Tolerant Lewis-Acid Catalyst. Bulletin of the Chemical Society of Japan 68, 1721-1730 (1995).

31. M. D. Levin, T. Q. Chen, M. E. Neubig, C. M. Hong, C. A. Theulier, I. J. Kobylianskii, M. Janabi, J. P. O'Neil, F. D. Toste, A Catalytic Fluoride-Rebound Mechanism for C( $\left.\mathrm{sp}^{3}\right)-\mathrm{CF}_{3}$ Bond Formation. Science 356, 1272-1275 (2017).

32. S. S. Chitnis, J. H. W. LaFortune, H. Cummings, L. L. Liu, R. Andrews, D. W. Stephan, Phosphorus Coordination Chemistry in Catalysis: Air Stable P(III)-Dications as Lewis Acid Catalysts for the Allylation of C-F Bonds. Organometallics 37, 4540-4544 (2018).

33. C. Bergquist, B. M. Bridgewater, C. J. Harlan, J. R. Norton, R. A. Friesner, G. Parkin, Aqua, Alcohol, and Acetonitrile Adducts of Tris(Perfluorophenyl)Borane: Evaluation of Bronsted Acidity and Ligand Lability with Experimental and Computational Methods. J. Am. Chem. Soc. 122, 10581-10590 (2000).

34. P. M. Zimmerman, Single-Ended Transition State Finding with the Growing String Method. J. Comput. Chem. 36, 601-611 (2015).

35. G. Stork, J. J. LaClair, Stereoselective Synthesis of Beta-Mannopyranosides via the Temporary Silicon Connection Method. J. Am. Chem. Soc. 118, 247-248 (1996).

36. J. T. Walk, Z. A. Buchan, J. Montgomery, Sugar Silanes: Versatile Reagents for Stereocontrolled Glycosylation Via Intramolecular Aglycone Delivery. Chem. Sci. 6, 34483453 (2015). 


\section{Acknowledgments:}

Prof. Pavel Nagorny, Prof. Nathaniel Szymczak, Prof. David Sherman (University of Michigan) are thanked for helpful discussions and Dr. Andrew Robertson is thanked for assistance with HPLC purifications. The authors thank the National Institutes of Health Common Fund Program in Glycoscience (U01GM125274) for support for this work. The University of Michigan has submitted a provisional patent application based on this work.

\section{Supporting information:}

General procedures are provided. 